\title{
Bounds on the Optimal Elasticity Parameters for a Snake
}

\author{
Ole V. Larsen ${ }^{1}$, Petia Radeva ${ }^{2}$ and Enric Marti ${ }^{2}$ \\ 1 Laboratory of Image Analysis, Aalborg University, Denmark \\ 2 Computer Vision Center, Autonomous University of Barcelona, Spain
}

\begin{abstract}
This paper develops a formalism by which an estimate for the upper and lower bounds for the elasticity parameters for a snake can be obtained. Objects different in size and shape give rise to different bounds. The bounds can be obtained based on an analysis of the shape of the object of interest. Experiments on synthetic images show a good correlation between the estimated behaviour of the snake and the one actually observed. Experiments on real X-ray images show that the parameters for optimal segmentation lie within the estimated bounds.
\end{abstract}

Keywords: snakes, elasticity parameters, segmentation.

\section{Introduction}

Snakes have several advantages compared to traditional segmentation techniques especially in case of noisy images and partly occluded objects [1, 2]. Snakes provide a model-based technique since ways exist to incorporate information about the object of interest into the snake formalism. An initial snake can be defined specifying the snake in accordance with the object of interest. Besides, it is possible by controlling the internal energy to constrain the smoothness of the snake. The smoothness required for an accurate segmentation is controlled by the elasticity parameters and is closely related to the shape of the object.

Despite the great interest in snakes, estimation of the elasticity parameters has not been given much attention previously. Samadani [5] dynamically estimates and adjusts the parameters to avoid instability in the deformation process. In contrast with his work our purpose is, based on knowledge about the size and shape of the object, to establish bounds on the parameters of elasticity that provide optimal smoothness of the snake.

Here we present a new formalism by which the upper and lower bounds on the elasticity parameters can be obtained through an analysis of a given sample of the object of interest, hereafter the object model. First, the theoretical foundation for the existence of bounds on the parameters is developed independently of the chosen snake implementation. Based on an implementation using the Finite Difference Method (FDM) formulas are then derived for calculating the bounds based on the shape of the object. At last, experimental results are presented to determine the correlation between the estimated behaviour of the snake and the one actually observed. 


\section{Parameters of elasticity}

A snake is an elastic curve $u(s)=(x(s), y(s))$ for which an energy function $E_{\text {snake }}$ is defined based on an internal energy $E_{\text {int }}$ and an external energy $E_{\text {ext }}$,

$$
E_{\text {snake }}=\int_{0}^{1} E_{\text {int }}(u(s))+E_{\text {ext }}(u(s)) d s .
$$

The internal energy is given by the sum of the membrane energy and the thinplate energy: $E_{\text {int }}(u)=\alpha E_{\text {membrane }}+\beta E_{\text {thin-plate }}=\alpha u^{\prime}(s)+\beta u^{\prime \prime}(s)$. The parameters $\alpha$ and $\beta$ are the parameters of elasticity. $\alpha$ controls the stretching and $\beta$ the bending of the snake curve.

The external energy is obtained from a potential field derived as the image gradient [2] or as a distance map of the edge points [1]. Without loss of generality we use the latter definition leading to the following expression, $E_{e x t}(u(s))=$ $P(x, y)=d(x, y)$, where $d(x, y)$ is the distance between pixel $(x, y)$ and the closest edge point. Minimizing the external energy the snake is attracted towards the edge points of the image. Minimizing the internal energy the shape of the snake is smoothed. The result of the segmentation is obtained when the snake detects a minimum of the total energy.

High values of the parameters of elasticity put great weight on the internal energies and consequently give a smooth curve. In general, we want the parameters to be as high as possible. It will decrease the risk of the snake being attracted to spurious noisy edges during its iterations [1]. However, if the parameters become too high, problems in terms of contour surpassing occur.

Let us consider a snake, $u_{c}$, of length $n$ placed in the potential valley corresponding to the contour of the object we are looking for. And let $\epsilon$ be some predetermined constant. The following definitions can be stated,

Definition 1 The minimum area around the contour valley containing all possible deformed versions, $u_{t}$, of snake $u_{c}$ in a distance less than $\epsilon$ to $u_{c}\left(\left\|u_{t}-u_{c}\right\|<\right.$ $\epsilon n)$ is called the $\epsilon$-neighbourhood of the contour valley.

Definition 2 Local surpassing is when the snake $u_{c}$ in the process of energy minimization moves locally out of the contour valley. Global surpassing is when the snake $u_{c}$ deforms to a snake outside an $\epsilon$-neighbourhood of the contour valley given a constant $\epsilon$.

An illustration of local and global surpassing is given in figure 1.

Definition 3 The set of maximum values of the elasticity parameters for a snake that does not give rise to any local surpassing is called local (surpassing) parameters. The set of maximum values that, given some $\epsilon$, does not give rise to any global surpassing is called global (surpassing) parameters.

The fact that the local parameters are the maximum parameters that do not give rise to any local surpassing from the contour means that the deforming 


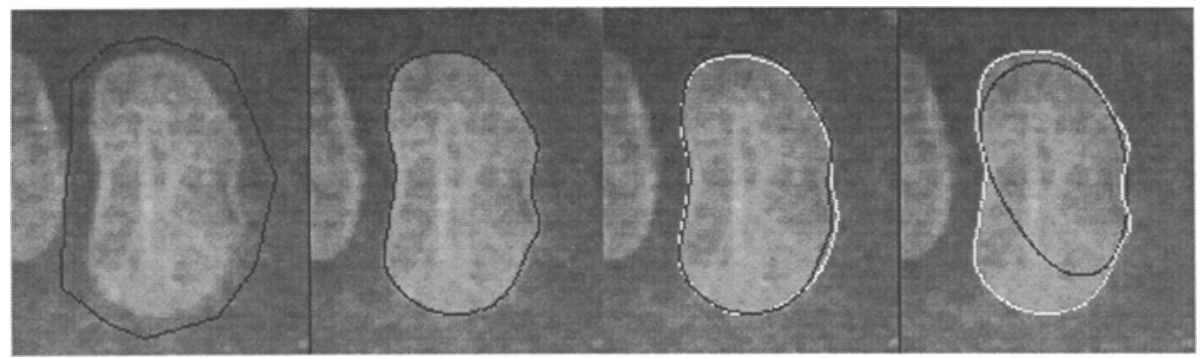

Fig. 1. Different situations for segmentation of a bone structure in a X-ray image; from left to the right: an initial snake, the snake shrunk to the contour, a local surpassing of the contour shown in white by the snake shown in black and a global surpassing by the snake.

snake by these parameters of elasticity will not get shapes more stretched neither bended than the represented in the object model. Due to the monotony property of the parameters of elasticity, stated in Lemma 1 and 2, each pair of parameters greater than the local parameters will not allow discontinuities in the snake not proper to the object model. Hence, the local parameters can be thought of as lower bounds on the parameters giving an optimal segmentation. The global parameters are the maximum values for which the displacement of the snake does not exceed some $\epsilon$-neighbourhood of the contour. The $\epsilon$-neighbourhood is to be determined by the user as the area around the contour where the snake does not lose its influence. The global parameters therefore determine the upper bounds on the parameters of elasticity.

Smoothing its shape the snake has a tendency to shrink itself. Based on the properties of the global parameters of elasticity it can be shown that the initial snake placed around the object is able to fall into an $\epsilon$-neighbourhood of the object contour. This paper therefore proposes an approach to determine the bounds by an analysis of the internal and external energies when the snake, from a position on the contour, deforms to surpass the contour.

\section{$2.1 \quad$ Fundamentals}

Before being able to determine formulas for calculating the bounds it is necessary to see some fundamental relations concerning certain properties of the parameters of elasticity. The fundamentals are stated without proofs, for more details see [3]. It should be emphasized that relations described in this section do not depend on any specific snake implementation, neither on any specific calculation of the potential field.

Let us consider the initial snake $u_{0}$, the snake $u_{c}$ shrunk to the contour valley and the snake $u_{t}$ converged from the snake $u_{c}$. Let $E_{\text {membrane }}^{c}, E_{\text {thin-plate }}^{c}$, $P^{c}, E_{m e m b r a n e}^{t}, E_{\text {thin-plate }}^{t}$ and $P^{t}$ be the membrane energy, the thin-plate energy and the potential energy of the snakes $u_{c}$ and $u_{t}$, respectively. 
Lemma 1. Let the parameters of elasticity $\left(\alpha^{\prime}, \beta^{\prime}\right)$ be such that the snake leaves the $\epsilon$-neighbourhood of the contour valley given some $\epsilon$. Then for any greater parameters of elasticity $\alpha \geq \alpha^{\prime}$ and $\beta \geq \beta^{\prime}$ the snake leaves the $\epsilon$-neighbourhood.

Lemma 2. Let the parameters of elasticity $\left(\alpha^{\prime}, \beta^{\prime}\right)$ be such that the snake remains in the $\epsilon$-neighbourhood of the contour valley given some $\epsilon$. Then for any smaller parameters of elasticity $\alpha \leq \alpha^{\prime}$ and $\beta \leq \beta^{\prime}$ the snake remains in the $\epsilon$-neighbourhood.

These lemmas define a kind of monotony of the parameters. They show that for a given parameter setting which is found to give local or global surpassing, any parameter value greater than the ones found will also cause surpassing.

Looking at differences in $E_{\text {int }}$ and $E_{\text {ext }}$ between $u_{c}$ on the contour and $u_{t}$ outside an $\epsilon$-neighbourhood we get the following theorem,

Theorem 3. The maximum pair of parameters of elasticity that retains the snake $u_{c}$ in an $\epsilon$-neighbourhood of the contour is given by the formulas,

$$
\alpha=\frac{P^{t}-P^{c}}{2\left(E_{\text {membrane }}^{c}-E_{\text {membrane }}^{t}\right)}, \quad \beta=\frac{P^{t}-P^{c}}{2\left(E_{\text {thin-plate }}^{c}-E_{\text {thin-plate }}^{t}\right)}
$$

where the snake $u_{t}$ is obtained from the snake $u_{c}$ and $\left\|u_{t}-u_{c}\right\|=\epsilon n$.

\subsection{Calculating the bounds of the optimal parameters of elasticity}

Given a specific shape model, the task now becomes one of determining the parameters not giving rise to local and global surpassing with respect to some constant $\epsilon$. They can be obtained from formulas (1) substituting the estimations of the energies of the snake $u_{c}$ placed in the contour valley, and its deformation $u_{t}$ in a distance $\epsilon$ from the contour. Without loss of generality we compute the parameters for the snake implementation by FDM and use the fact that the potential is generated as a distance map of the edge points of the original image. In order to simplify our calculus we ensure that the snake pixels are evenly distributed on the curve in each iteration of the snake movement.

The energies of $u_{c}$ can be estimated by the initial snake $u_{0}$ and the object model [3]. In order to calculate the global parameters we have to estimate the deformation of the snake $u_{c}$ in a distance $\epsilon$. In [3] we have shown that the deformation of a snake in a given distance can be approximated by the deformation of a circle with the same length. Substituting in (1) the energies of the circle and its deformation after global surpassing in distance $\epsilon$, we get,

$$
\alpha_{g l}=\frac{k n^{2}}{8 \pi(n-\epsilon k \pi)}, \quad \beta_{g l}=\frac{k n^{4}}{32 \pi^{3}(n-\epsilon k \pi)}
$$

where $k=\frac{u_{0}}{u_{c}}$ is the accumulation rate of the initial snake in the contour valley. 
For the case of the local parameters we use the fact that local surpassing firstly occurs in the most curved segment of the snake. From formulas (1) we get,

$\alpha_{l o c}=\frac{\theta\left\lceil\frac{m}{2}\right\rceil k^{2}}{2 m\left(1-2 k^{2}(r+\epsilon)^{2}\left(1-\cos \frac{\mu}{m}\right)\right)}, \quad \beta_{l o c}=\frac{\theta\left\lceil\frac{m}{2}\right\rceil r^{2}}{2 m\left(1-4 r^{2}(r+\epsilon)^{2}\left(1-\cos \frac{\mu}{m}\right)^{2}\right)}$

$\theta=\sqrt{r^{2}+(r+\epsilon)^{2}-2 r(r+\epsilon) \cos \frac{\nu-\mu}{2}}-\epsilon, \mu=\arccos \left(\frac{\epsilon(2 r+\epsilon)+r^{2} \cos \left(m \arccos \left(1-\frac{1}{2 r^{2}}\right)\right)}{(r+\epsilon)^{2}}\right)$ where $m, \nu$ and $r$ are the length, the angle, and the radius of the most curved segment of the snake $u_{c}$.

\section{Results and discussion}

Two lines of experiments have been conducted. One based on synthetic images and one based on real X-ray images of bone structures.

The test on synthetic images investigated to what degree the calculated local and global surpassing parameters gave the expected surpassings if a snake was initialised with the values. Six different types of objects have been used: circle, octagon, hexagon, square, rectangle and triangle, each represented with scaled versions having length $100,200, \ldots, 500$ pixels. For each object the values of $\alpha$ and $\beta$ from formulas (1) were computed given $\epsilon=1.5$. The experiments showed that the precision by which the estimated behaviour fitted the observed behaviour did not depend on the length of the snake. Furthermore, it showed that for 5 out of 6 objects the converged snake had a distance from the contour in the interval $[1.5 ; 2.0]$, with $\epsilon$ being 1.5 . Only for the triangle the distance was as high as 3.5 . From the experiments it can be concluded that the surpassings estimated based on parameters calculated from the object model are similar to the surpassings observed when a snake is used to converge around the object, with a slight tendency to underestimate the actual surpassings. Objects with sharp corners tend to have less similar surpassings. By letting snakes converge with parameters from the interval $[0.5 ; 2.0]$ times the estimated $(\alpha, \beta)$, it was observed that the distance between the contour and the converged snake increased as the values increased. This supports the monotony estimated in Lemma 1 and 2.

The test on real images comprised 15 images of hand radiographs. For each bone we used as an initial snake a model with similar structure but larger in size. Using local surpassing parameters it was observed that depending on the difference between the initial snake and the object contour in some cases the snake moved correctly to the contour (Fig. 3a (left)). In other cases the snake remained outside the contour due to attraction by near edge points (Fig. 3b(left)).

In accordance to our observations the global parameters assure us that the snake will surpass the contour going out in some distance with predetermined magnitude (Fig. 3a (right) and Fig. 3b (right)). In this way any snake detention in a local energy minimum before arriving to the contour will be avoided. Due to the monotony property of the elasticity parameters we can state that the parameters of the optimal segmentation belong to the intervals determined by the local and global parameters. 


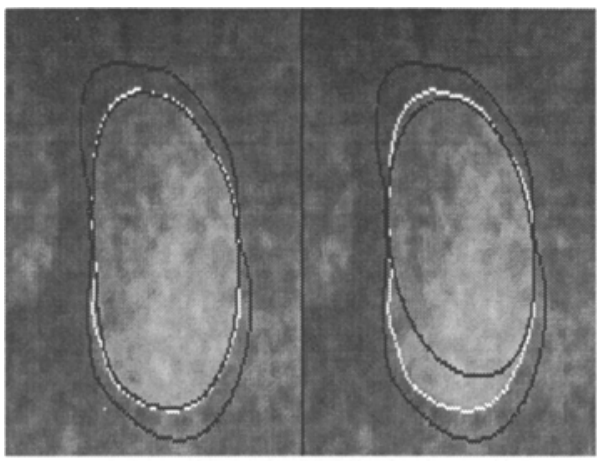

a

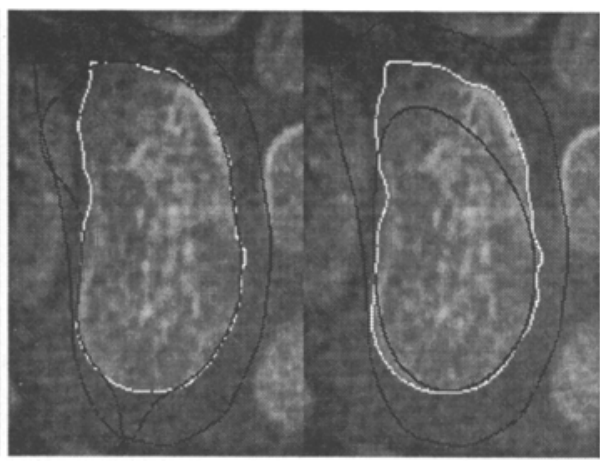

b

Fig. 2. Two different situations of using local (left) and global (right) parameters. The white curve is the contour of the objects. The outer curve represents the initial snake and the inner curve the resulting converged snake.

\section{Conclusion}

Based on the concept of local and global surpassing we have defined some local and global surpassing parameters which serve as lower and upper bounds on the elasticity parameters of a snake in terms of segmentation of a given object. Formulas are presented by which an estimate of the parameters bounds can be calculated for a given object model. Experiments show a good correlation between the estimate and results obtained on synthetic images. Experiments on real $\mathrm{X}$-ray images show that optimal segmentation is only possible by parameters within the bounds defined in this paper. By establishing bounds on the parameters the search space of the values giving the best segmentation is limited. In [4] we propose guidelines for non-snake experts to determine the optimal parameters of elasticity based on an automatic inspection of the object models.

\section{References}

1. I. Cohen, L. D. Cohen, and N. Ayache. Using deformable surfaces to segment 3-d images and infer differential structures. CVGIP: Image Understanding, 56(2):242263, 1992.

2. M. Kass, A. Witkin, and D. Terzopolous. Snakes: Active contour models. In International Conference on Computer Vision, London, pages 259-268, 1987.

3. O.V. Larsen and P. Radeva. Calculating the bounds on the optimal parameters of elasticity for a snake. Technical report, Laboratory of Image Analysis, Aalborg University, Denmark, December 1994.

4. O.V. Larsen, P. Radeva, and E. Martí. Guidelines for choosing optimal parameters of elasticity for snakes. In Proceedings from CAIP'95 - International Conference on Computer Analysis and Image Processing (Accepted), 1995.

5. R. Samadani. Adaptive snakes: Control of damping and material parameters. In SPIE Geometric Methods in Computer Vision, volume 1570, 1991. 